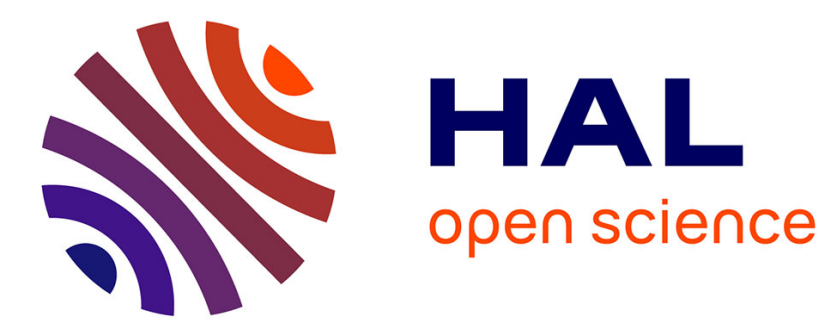

\title{
Application of a Bayesian approach for exploring the impact of syllable frequency in handwritten picture naming
}

\author{
Cyril Perret, Clara Solier
}

\section{To cite this version:}

Cyril Perret, Clara Solier. Application of a Bayesian approach for exploring the impact of syllable frequency in handwritten picture naming. Journal of Cognitive Psychology, In press, pp.1-14. 10.1080/20445911.2022.2029459 . hal-03545161

\author{
HAL Id: hal-03545161 \\ https://hal.science/hal-03545161
}

Submitted on 25 Feb 2022

HAL is a multi-disciplinary open access archive for the deposit and dissemination of scientific research documents, whether they are published or not. The documents may come from teaching and research institutions in France or abroad, or from public or private research centers.
L'archive ouverte pluridisciplinaire HAL, est destinée au dépôt et à la diffusion de documents scientifiques de niveau recherche, publiés ou non, émanant des établissements d'enseignement et de recherche français ou étrangers, des laboratoires publics ou privés. 


\section{Application of a Bayesian approach for exploring the impact of syllable frequency in handwritten picture naming}

Journal of Cognitive Psychology, http://dx.doi.org/10.1080/20445911.2022.2029459 Accepted 2022-1-11

Cyril Perret $^{1} \&$ Clara Solier $^{1}, 2$

${ }^{1}$ Univ. Poitiers, CeRCA (CNRS UMR 7295), Poitiers, France

${ }^{2}$ Univ. Poitiers, MSHS (USR 3565), Poitiers, France

Corresponding author:

Cyril Perret

Centre de Recherche sur la Cognition et l'Apprentissage (CeRCA UMR 7295 - CNRS)

Université de Poitiers

MSHS Bâtiment A5

5, rue Théodore Lefebvre

TSA 21103

F-86073 Poitiers Cedex 9

cyril.perret@univ-poitiers 


\begin{abstract}
Adult studies in the fields of neurolinguistic and mental chronometry suggest that the syllable plays a functional role in handwritten word production. These studies support the hypothesis of a syllabified orthographic representation stored in the graphemic buffer. However, there remains the question of the cognitive mechanisms involved in this encoding of orthographic representations and, in particular, that of the processes related to the syllable. In the study reported here, we tested the hypothesis of an orthographic mental syllabary in long-term orthographic memory by exploring the impact of syllable frequency on handwritten latencies. Thirty participants handwrote the labels of one hundred and fifty images. Bayesian analyses indicated that the data support an absence of effect of syllable frequency. We propose an alternative hypothesis to the syllabary to account for the results in the literature. This respects the constraint of an absence of effect of syllable frequency in handwritten word production.
\end{abstract}

Keywords: syllable frequency; reaction times; handwritten picture naming; Bayesian analyses 


\section{INTRODUCTION}

Over the last thirty years, an increasing number of studies have investigated the cognitive processes involved in handwritten word production in adults (for a review, see Perret \& Olive, 2019). Handwriting a word involves two stages: the preparation of a verbal response following presentation of a stimulus and the motor execution of this response. Whatever the modality of the stimulus (visual, auditory, conceptual), the preparation of the handwritten response involves two levels of processing (e.g., Planton et al., 2013; Purcell et al., 2011; Roux et al., 2013). The central level brings together the cognitive processes involved in accessing abstract orthographic representations, which minimally encode the identity and order of letters. The spelling codes are recovered by integrating information from two processing routes (Bonin et al., 2015; Rapp et al., 2002; Rapp et al., 2016): the lexical route retrieves information from orthographic longterm memory or orthographic lexicon (O-LTM); the sub-lexical route is based on phonologyto-orthography conversion (POC) procedures. The retrieved abstract orthographic representation is kept active in orthographic working memory (O-WM), or graphemic buffer (Rapp et al., 2016). The second level of processing includes all the peripheral processes. Based on the information transmitted by $\mathrm{O}-\mathrm{WM}$, a set of processes transforms the abstract orthographic codes into gestures directly executable by the motor system (Ellis, 1988; van Galen, 1991). This involves the choice of the allograph for each letter, access to the graphic motor programmes and the planning of gestures before motor execution begins.

One question that studies on handwritten word production have tried to answer relates to the linguistic format of the representations involved in orthographic response preparation. The syllable is one potential candidate. A number of studies concur that abstract orthographic representations stored in $\mathrm{O}-\mathrm{WM}$ have a multidimensional organisation, with one of these dimensions corresponding to the syllabic structure (e.g., Hess et al., 2019; Kandel et al., 2011; Lambert et al., 2008; Sausset et al., 2012). Nevertheless, these studies do not allow us to come 
to any direct decision regarding the hypothesis that orthographic syllables are stored in O-LTM (i.e. an orthographic mental syllabary, Kandel et al., 2011). The aim of the present study was to test this hypothesis by exploring the influence of orthographic syllable frequency on handwritten response preparation time, i.e., handwriting latencies.

One of the most frequently cited neurolinguistic arguments in favour of the role of the syllable in handwritten production is the performance pattern of the patient reported by Caramazza and Miceli (1990). Among the errors produced by LB, some were constrained by the syllabic structure of the to-be-produced item, such as letter shifts within the same syllable (e.g., tornava -> tronava) or simplification of the syllabic structure through the omission of a letter (e.g., presso -> pesso). Since LB was suffering from a graphemic buffer disorder (Caramazza, Miceli, Villa, Romani, 1987), it is possible to account for this pattern of performance in terms of the syllabic organisation of the stored representations in O-WM (see, however, Jónsdóttir et al., 1996; McCloskey et al., 1994; Ward \& Romani, 2000). Errors involving a position change of geminate letters (e.g., ll, rr, ee) produced by the patient HE (Badecker, 1996) are also consistent with the proposal made by Caramazza and Miceli (1990). Like LB, HE suffered from a graphemic buffer disorder (McCloskey et al., 1994). In English, geminate letters are illegal at syllable onset, whereas they are legal in rime. The way in which the syllabic structure constrains the possible position exchanges of the doublet should therefore allow the error neddle for needle but not pparot for parrot. Badecker (1996) showed that 99\% of the geminate letter position exchanges produced by HE led to errors whose syllabic structure remained legal.

Studies on adults without language impairment using on-line measures of handwritten production have explored the impact of an item syllable number on latencies. Lambert et al. (2008, see also Lambert et al, 2015) asked participants to copy bi-, tri-, and quadri-syllabic items three times. The number of syllables influenced the latencies of the second (time between 
the end of the first copy and the beginning of the second one, L2) and the third copy (L3) of the words. According to Lambert et al. (2012), L2 and L3 reflect only the processes underlying handwriting preparation itself, without any influence of visual perception processing. These processes include all peripheral processing levels. Regarding central processes (Rapp et al., 2016), Lambert et al.'s (2008) results suggest that access to orthographic codes in O-LTM and POC procedures occur only during L1 because the lexical frequency and lexicality effects were significant only for the first copy latency. According to Lambert et al. (2008), a rehearsal process in which the syllable is the processing unit makes it possible to maintain the orthographic representation active in O-WM before the second and third copy of the word (see also Service \& Turpeinen, 2001).

The syllabic structure has also been reported to have an influence on the execution of the graphic trace. Kandel et al. (2006) found that the inter-letter duration was longer when a bigram (e.g., ac) corresponds to a syllabic boundary (e.g., tra.ceur $)^{1}$ than when this is not the case (e.g., trac.tus). Similar results have been reported with different types of tasks (dictation, copying, picture naming), in both upper and lower-case handwriting, in typewriting and in several alphabetic languages (Alvarez et al., 2009; Bogearts et al., 1996; Hess et al., 2019; Kandel, et al., 2006, 2011; Kreiner et al., 2008; Sausset et al., 2012, 2013; Service \& Turpeinen, 2001; Weingerten et al., 2004; Zesiger et al., 1994). A conception based on parallel processing at the central and peripheral levels (e.g., Kandel et al., 2011; Olive, 2014; Roux et al., 2013) makes it possible to account for syllabic boundary effects. As cognitive resources are limited, they are shared between the different levels of processing at any given point in time (Olive, 2014). Crossing a syllabic boundary requires additional processing at the central level, thereby increasing resource consumption. As a result, there are fewer resources available for peripheral

\footnotetext{
${ }^{1}$ The dot refers to the syllabic boundary.
} 
processing, resulting in a slowdown. An increase in the inter-letter space writing time corresponding to a syllabic boundary is then observed.

The influence of the number of syllables on the preparation time for the second and third copy and the modification of the handwriting dynamics when crossing a syllabic boundary are arguments in favour of a functional role of the syllable in handwritten production. However, it may seem contradictory that syllables can be processed either during the preparation (copy latencies) or during the execution of the handwritten response (syllabic boundary crossing). To account for this pattern of results, Sausset et al. (2012) proposed the existence of a link between the need for cognitive resources and the occurrence of syllable effects either before or during handwriting. Using Lambert et al.'s (2008) triple-copy paradigm, these authors asked their participants to produce pairs of items similar to those used by Kandel et al. (2006, trac.tus versus tra.ceur). They also manipulated the level of attentional resources involved in peripheral processing by varying the requirements in terms of graphomotor characteristics (Olive \& Kellogg, 2002): writing in lower case, upper case, large capitals and, finally, large capitals without visual feedback (using a pen with a dry point). Sausset et al. (2012) showed that the syllable number effects on L2 and L3 (Lambert et al., 2008) disappear when graphomotor constraints increase, whereas the influence on the motor characteristics at a syllabic boundary (Kandel et al., 2006) increase. According to Sausset et al. (2012), syllable processing is completed before copying (L2 and L3) when the cognitive resource demand of peripheral processing is low. Alternatively, when the attentional demand required for peripheral processing increases, writers may programme their production syllable by syllable. Combining this proposal with that of Lambert et al. (2008), it is possible to hypothesise that the increase in demand for resources due to graphomotor constraints prevents the rehearsal process from occurring. The orthographic codes would therefore be accessed in O-LTM for each copy. 
Figure 1. Model describing the linguistic units involved in manuscript production, adapted from Kandel et al. (2011).

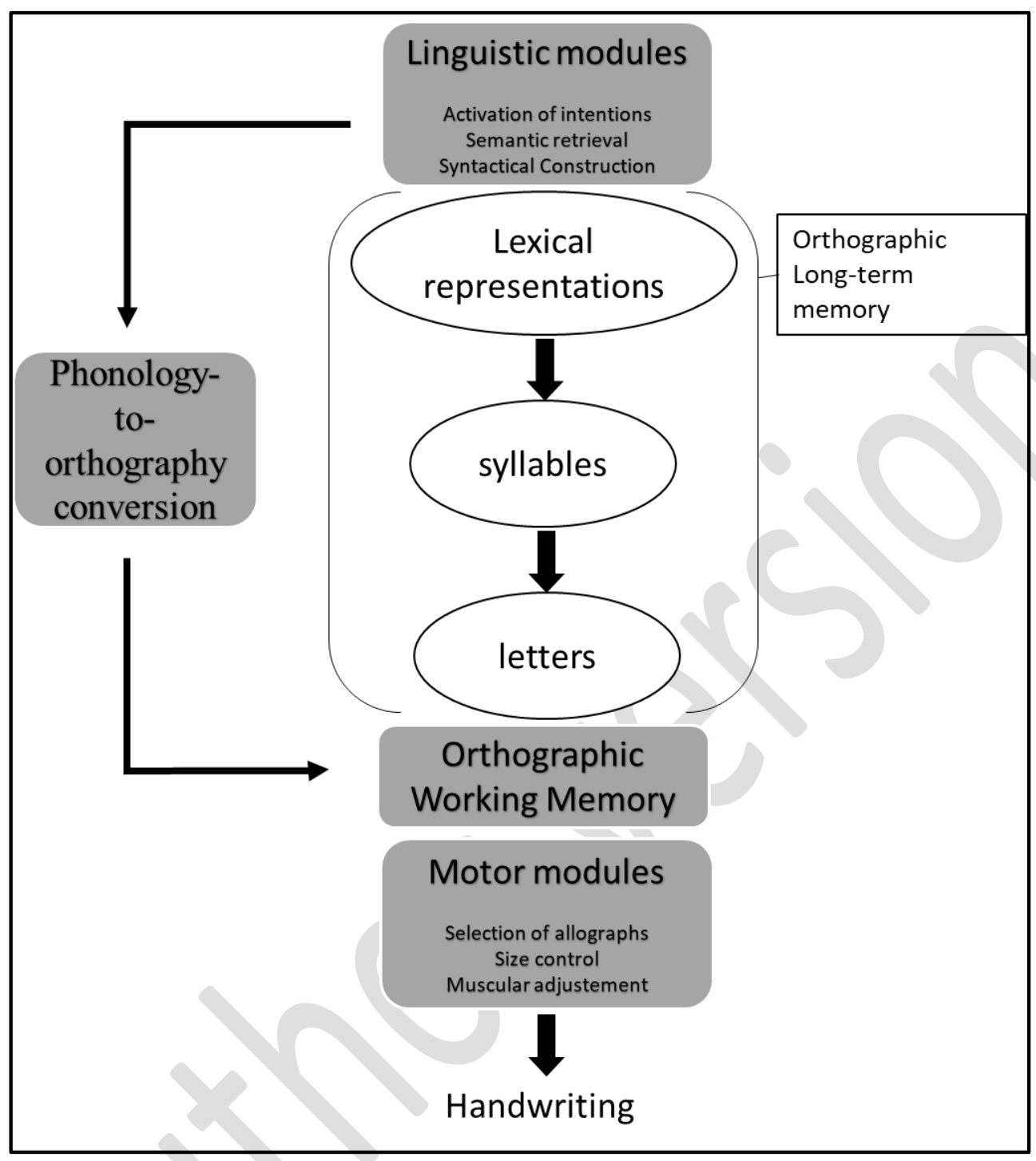

Taken together, neurolinguistic and mental chronometry studies support the idea of a functional role for the syllable in O-WM. This linguistic unit is one of the organisational dimensions of the orthographic representation maintained active in O-WM. Neurolinguistic and mental chronometry studies do not, however, make it possible to come to any decision regarding the processes underlying the recovery of this syllable-based orthographic representation. To shed some light on these issues, Kandel et al. (2011) proposed a model integrating syllables as units of orthographic wordform which encode processing. In handwritten production, it is widely accepted that orthographic wordform encoding involves at least two levels of 
representation (Rapp \& Fisher-Baum, 2014). A first level corresponds to the morphological structure of the word. Lexical access begins with the retrieval in O-LTM of the different morphemes constituting the word to handwrite. The letters/graphemes ${ }^{2}$ correspond to linguistic units of the second level of representation. Letters are abstract entities, lacking visual or motoric form, stored in O-LTM. According to Rapp \& Fisher-Baum (2014), the O-WM is involved in selecting letters in the correct order. The proposal by Kandel et al (2011) aims to include a third level of representations between the two described above. The representations of this third level would have a syllabic format and would be stored in O-LTM (Figure 1). The selection of a morpheme would trigger access to syllables. The latter would then drive access to the letters, thus specifying the syllabic structure of the lexical representation maintained active in O-WM. Our aim in this work is to test this proposal in an experimental setting.

The conception of access to the orthographic wordform proposed by Kandel et al. (2011) accounts for the syllable effects reported in the literature. As the process of retrieving a syllable from the orthographic mental syllabary is costly, the crossing of a boundary between two syllables impacts processing (Kandel et al., 2006, 2011). This results in a greater inter-letter duration when a bigram (e.g., ac) corresponds to a syllabic boundary (e.g., tra.ceur) than when it does not (e.g., trac.teur). Moreover, as writers plan their production syllable by syllable then, when the demand for resources by peripheral processing levels increases (Sausset et al., 2012), the operation of accessing the orthographic mental syllabary before each syllable impacts the amount of resources available for the other activities which are being performed. This is reflected by a syllabic boundary effect in conditions where large capitals have to be produced with or without visual feedback (Sausset et al., 2012). Finally, the hypothesis proposed by Kandel et al (2011) makes it possible to account for the effects of the number of syllables (e.g., Lambert et al., 2008) and the observed syllabic constraints on patient errors (e.g., Badecker,

\footnotetext{
${ }^{2}$ The grapheme corresponds to the graphic realisation of a phoneme and can be composed of several letters. Here we use these two conceptions interchangeably.
} 
1996; Caramazza \& Miceli, 1990) because the orthographic mental syllabary is the source of the syllabic dimension of the orthographic representation present in the graphical buffer.

An influence of syllable frequency on handwriting latencies would be an argument in favour of the orthographic mental syllabary hypothesis. A linguistic unit that occurs often in a language is regularly processed by the cognitive system. This processing increases its availability in long-term memory. At the operational level, a decrease in response preparation time for items with high-frequency representations should be observed. One example is the observed influence of lexical frequency on handwritten latencies (Bonin et al., 1998; Bonin et al., 2016; Zhang \& Wang, 2014). According to Kandel et al.'s (2011) model, syllable frequency should influence handwriting latencies. A syllable frequency effect on the dynamic aspect of writing has been reported by Afonso and Alvarez (2011). Participants produced trisyllabic items on the basis of which the frequency of the second syllable was manipulated. The syllable frequency affected the inter-letter intervals of the second syllable: the higher the frequency was, the shorter the duration. According to Afonso and Alvarez (2011), the results suggest that the letters of high-frequency syllables are more easily and quickly associated than those of lowfrequency syllables. However, it should be noted that the influence of syllable frequency on the dynamic aspects of adult handwriting was not replicated by Kandel et al. (2011). Zhang and Wang (2014) tested the influence of syllable frequency on latencies in Chinese. Participants had to handwrite picture names three times. Syllable frequency and lexical frequency were orthogonally manipulated. The main effect of lexical frequency was clearly observed, whereas the syllable frequency effect was marginal, occurring only during the second copy of the picture name. This near absence of effect is, to the best of our knowledge, the only attempt to show an influence of syllable frequency on handwritten latencies.

The aim of the present study was to test the hypothesis of an influence of orthographic syllable frequency on the response preparation processes. Thirty undergraduate students had to 
handwrite the names of 150 pictures. We first ran a multiple linear regression model including the experimental factors used in the literature (Bonin et al., 2002; Bonin et al., 2015; Perret \& Bonin, 2019; Perret \& Laganaro, 2013). We then tested the impact of syllable frequency. This variable was operationalised in two different ways. First, we used an average of the frequency of the syllables of a word. For instance, for a trisyllabic word such as "ananas" [pineapple], the frequency of the three syllables was added and the resulting value divided by three. This operationalisation of syllable frequency, however, is based on an implicit assumption that the three syllables of the word are recovered in O-LTM before writing begins. Works on inconsistency/irregularity in handwritten production have suggested instead that writing begins before the full set of orthographic codes is available (e;g., Bonin et al., 2001; Kandel \& Perret, 2015; Roux et al., 2013). Bonin et al. (2001) showed, for example, that the presence of an inconsistency/irregularity influences the initialisation latencies of monosyllabic words only when this difficulty is in the initial position. In a second step, we then used the frequency of the first syllable as operationalisation of the syllable frequency. Our study being, to the best of our knowledge, the first to address this question, the syllable frequency effect was examined using a Bayesian approach rather than a frequentist one.

\section{METHOD}

\section{Participants}

Thirty undergraduate students (10 men; aged, 17-28; mean: 22.57) from Neuchâtel University (Switzerland) took part in this experiment ${ }^{3}$. They were right-handed French native speakers with no visual, motor or language impairments. They received course credits for their participation. The study complied with the 2008 Helsinki Declaration. Participants provided written informed consent.

\footnotetext{
${ }^{3}$ The data presented here were collected in 2009 during the first author's post-doctoral position at the University of Neuchâtel (Switzerland).
} 


\section{Stimuli}

One hundred fifty drawings were selected from two French picture databases (Alario \& Ferrand, 1999 and Bonin, Peereman et al., 2003) ${ }^{4}$. The following nine properties were obtained for each stimulus (Table 1).

Table 1. Descriptive statistical for the nine factors

\begin{tabular}{ccccccccc}
\hline Factors & Mean & Q1 & Median & Q3 & S.D. & Min & Max & Skewness \\
\hline NA (h) & 0.28 & 0.00 & 0.00 & 0.34 & 0.42 & 0.00 & 1.87 & 1.86 \\
IA & 3.67 & 3.20 & 3.72 & 4.25 & 0.74 & 1.23 & 4.90 & -0.63 \\
Ivar & 2.75 & 2.34 & 2.64 & 3.12 & 0.64 & 1.33 & 4.70 & 0.67 \\
AoA & 2.37 & 1.85 & 2.28 & 2.88 & 0.59 & 1.12 & 4.62 & 0.51 \\
LogFreq & 1.00 & 0.59 & 0.99 & 1.32 & 0.78 & 0.03 & 2.77 & 0.61 \\
NbLett & 5.85 & 4.00 & 6.00 & 7.75 & 1.98 & 2.00 & 10.00 & 0.09 \\
Fam & 3.06 & 2.13 & 3.07 & 4.02 & 1.09 & 1.03 & 4.97 & 0.02 \\
VC & 3.01 & 2.39 & 3.00 & 3.58 & 0.89 & 1.00 & 5.00 & 0.04 \\
LogMSyllF & 2.21 & 1.68 & 2.36 & 2.74 & 0.84 & -0.27 & 4.10 & -0.27 \\
LogFSyllF & 2.26 & 1.51 & 2.33 & 2.95 & 1.02 & -0.27 & 4.4 & -.03 \\
\hline
\end{tabular}

Note. $Q 1: 25^{\text {th }}$ percentile; $Q 3: 75^{\text {th }}$ percentile; S.D.: Standard Deviation; Min: Minimum; Max; Maximum; $N A(h)=$ Name Agreement, $\mathrm{h}$-statistic measures; IA = Image Agreement; Ivar = Image Variability; $A o A=$ Age of Acquisition; LogFreq = Natural logarithm of lexical frequency; NbLett $=$ Number of letters; Fam = Conceptual Familiarity; $V C=$ Visual Complexity; $L o g M S y l l F=$ Natural logarithm of syllable frequency; $\log F$ SyllF = Natural logarithm of frequency of the first syllable

The age at which the words were learnt (Age of Acquisition, AoA) was included, using adult ratings. The three picture variables reported by Bonin et al. (2002, 2015; Perret \& Laganaro, 2013; Perret \& Bonin, 2019) as being significant predictors of handwritten latencies

\footnotetext{
${ }^{4}$ The full list of stimuli and their values for each variable are available on OSF (http://osf.io/ gazf3).
} 
were included: Name Agreement (hNA), Image Agreement (IA), Image Variability (Ivar). We also included Conceptual Familiarity (Fam), Visual Complexity (VC), Lexical Frequency (LogFreq) and the number of letters (NbLett). The values for these eight factors were taken from two French databases (Alario \& Ferrand, 1999; Bonin et al., 2003). We used the measures of Lexical Frequency taken from LEXIQUE2 (New, Pallier, Brysbeart, \& Ferrand, 2004) transformed with natural logarithm. Syllable frequency values were obtained from the Chetail and Mathey (2010) database. Picture naming concerned mono-, bi-, and tri-syllabic words. Two measures of syllabic frequency were used (Table 1). The mean syllable frequency (LogMSyllF) was then computed for each word (e.g., Laganaro \& Alario, 2006; Perret et al., 2014). This was the average of the frequencies of each syllable of the word. The second measure was the frequency of the first syllable of the word (LogFSyllF).

\section{Procedure}

The participants were tested individually in a soundproof room where they were seated approximately $60 \mathrm{~cm}$ in front of the screen. There was no phase of familiarisation with the picture names ${ }^{5}$. The 150 pictures were presented randomly, preceded by three warm-up items. The DMDX software (Forster \& Forster, 2003) presented the trials and recorded the response latencies. The experimental trials had the following structure: a ready signal "+" appeared on the screen for $500 \mathrm{~ms}$, immediately followed by the picture. The drawing was presented in reverse video mode (white lines on black screen) in a constant size of $9.5 * 9.5 \mathrm{~cm}$ and remained visible on screen for $2000 \mathrm{~ms}$ if no answer was detected by the graphic tablet. The next trial began after $4000 \mathrm{~ms}$. The handwriting latency (RTs) was defined as the time that elapsed between the presentation of the picture and the first contact detected by the graphic tablet.

\footnotetext{
${ }^{5}$ As pointed out by Perret and Laganaro (2013), the familiarization phase seems to have consequences for the reported effects and more particularly for Name Agreement effects.
} 
The participants were told that they would see pictures and that they should write down (lowercase) the picture names as rapidly and as accurately as possible on a graphic tablet (WACOM UltradPad A5) using an inking contact pen (SP-401). A white sheet on a graphic tablet made it possible to gather the handwritten responses. Ninety-two 5-cm-long response lines were distributed evenly across four columns on each sheet. Moreover, the participants had to write down ToT (Tip-of-the-Tongue) when they recognised the picture but were not able to retrieve the name and DKO (don't know object) when they did not recognise the object. To avoid variability in the positioning of the pen, the participants were asked to position the stylus directly above the start of the line. They sat with the stylus right above the tablet so that the latency was the time taken to make contact after picture onset. There was a short break after every fifty trials. This allowed the experimenter to change the sheet on the graphic tablet. The experimental session lasted approximately thirty minutes.

\section{Statistical analyses}

We attempted to test the influence of syllable frequency on handwritten picture naming latencies (RTs) all other things being equal. We ran a multiple simultaneous regression model with handwriting latencies as dependent variable and with the eight experimental factors used in Bonin et al. (2002, 2015 see also Perret \& Laganaro, 2013; Perret \& Bonin, 2019). A Bayesian approach was favoured to the classical frequentist one because only very few studies have explored the influence of syllable frequency on handwriting latencies. Only Zhang and Wang (2014) reported a trend effect. It therefore seems equally important to conclude with regard to $\mathrm{H}_{0}$ (absence of a syllable frequency effect) as $\mathrm{H}_{1}$ (presence of a syllable frequency effect). Beyond the estimation of the model's parameters based on hypotheses on prior distribution (see below), the Bayesian approach makes it possible to calculate a ratio between two conditional probabilities. Let $\mathrm{H}_{1}$ and $\mathrm{H}_{2}$ be the two hypotheses and $\mathrm{D}_{\text {ata }}$ the data obtained in a study. The Bayes Factor (BF hereafter) then corresponds to the ratio between the likelihood 
of $\mathrm{H}_{1}$ given the data, $\mathrm{P}\left(\mathrm{H}_{1} \mid \mathrm{D}_{\text {ata }}\right)$ and the likelihood of $\mathrm{H}_{2}$ given the data, $\mathrm{P}\left(\mathrm{H}_{2} \mid \mathrm{D}_{\text {ata }}\right)$. Jeffreys (1961) proposed a classification of the BF values. If they are greater than 3 or less than $1 / 3$, this suggests a higher probability of $\mathrm{H}_{1}$ or $\mathrm{H}_{0}$, respectively; little evidence is provided either way if BF is between 1/3 and 3 (Dienes, 2016; Jeffreys, 1961) ${ }^{6}$.

The data as well as the script of the analyses in R-software are available on OSF (http://osf.io/gazf3). The data were fitted with a simultaneous linear mixed-effects model (Pinheiro \& Bates, 2000; Snijder \& Bosker, 1999) with the R software (version 3.6.1). The model included the eight experimental factors (Table 1) for population-level parameters and by-item adjustment on intercept, and by-participant adjustment on both the intercept and slopes for the group-level parameters. Analyses were run with the "brms" package (Bürkner, 2017, 2018). This package allows both the population- and group-level parameters to be estimated using a Bayesian approach based on Stan (Stan Development Team, 2017). The Bayesian approach makes it necessary to define the prior distribution of each model's parameters. With regard to the regression parameters at population level ( $\beta$ parameters), analyses were conducted with normal prior with 0 as mean and 100 as standard deviation $(\mathscr{N}(0,100))$. Three things have to be specified concerning regression parameters at group level. First, each grouping factor has a standard deviation parameter. This requires by-participant and by-item adjustment on intercept and by-participant adjustment on both intercept and the slope for the eight factors. A half-Cauchy prior distribution was used for each parameter (Gelman, 2006). Secondly, correlations between the group-level effects need to be included. Following Bürkner (2018), the LKJ-correlation prior with parameter $\zeta=2$ was used. Thirdly, it is necessary to address the question of the benefit of including the by-participant adjustments on the slopes and the correlations between group-level effects. To explore this issue, the models were compared using

\footnotetext{
${ }^{6}$ There is a large body of literature on Bayesian analyses. Readers who want to know more about these analyses can consult Kruschke's (2011) book and a recent special issue of Psychonomic Bulletin \& Review (Vandekerckhove et al., 2018).
} 
leave-one-out cross-validation (Vehtari et al., 2017). If there was little benefit in adding a group-specific coefficient, this was excluded from the final model.

Once the final model was fitted ${ }^{7}$, we computed two Bayes factors for each populationlevel parameter (Kass \& Raftery, 1995). The first ratio allowed us to explore whether the inclusion of an experimental factor increased the fit of the model, i.e., the bilateral hypothesis. $\mathrm{BF}_{10}$ was the ratio $\mathrm{P}\left(\mathrm{H}_{1} \mid \mathrm{D}_{\text {ata }}\right) / \mathrm{P}\left(\mathrm{H}_{0} \mid \mathrm{D}_{\text {ata }}\right)$, with the experimental factor being included in $\mathrm{H}_{1}(\beta$ $\neq 0$ ) and not in $\mathrm{H}_{0}$, (i.e., the null hypothesis, $\beta=0$ ). When the $\mathrm{BF}_{10}$ was greater than 3 (Dienes, 2016; Jeffreys, 1961), a second BF made it possible to test the direction of the relationship between the experimental factor and the handwriting latencies (i.e., the unilateral hypothesis). For instance, the theoretical relationship between age-of-acquisition and handwriting latencies is positive $(\beta>0)$, i.e., the later the word is acquired, the longer the handwriting reactions. $\mathrm{BF}_{\text {hyp }}$ was the ratio $\mathrm{P}\left(\mathrm{H}_{1} \mid \mathrm{D}_{\text {ata }}\right) \mathrm{P}\left(\mathrm{H}_{2} \mid \mathrm{D}_{\text {ata }}\right)$, where for $\mathrm{H}_{1} \beta>0$ and for $\mathrm{H}_{2} \beta \leq 0$. The beta estimation $(\beta)$, the standard deviation of the posterior distribution, the two-sided $95 \%$ credible intervals, and two Bayes Factors $\left(\mathrm{BF}_{10}\right.$ and $\left.\mathrm{BF}_{\mathrm{hyp}}\right)$ were determined for each experimental factor. The Bayesian R-squared was computed for the simultaneous linear regression model (Gelman, Goodrich, Gabry, \& Vehtari, 2019).

Finally, the influence of syllable frequency on handwriting latencies was tested. We used a model comparison approach. First, a reference model was specified ${ }^{8}$. The simultaneous linear regression described above served as a starting point. In the model, we retained only those factors for which the $\mathrm{BF}_{10}$ was greater than 3. A new regression was performed on the reference model by adding one of the syllable frequency measures, i.e. the augmented model. The a posteriori distribution was obtained for the reference model and for the augmented model. The

\footnotetext{
${ }^{7}$ Posterior distributions were estimated from 3000 iterations. The first 1000 made it possible to fit the model. 4 MCMC allowed us to obtain 8000 posterior samples. Lastly, the NUTS sampler behaviour (Bürkner, 2017) was monitored with a delta value .80 for the regression model and .95 for the syllable frequency test.

${ }^{8} \mathrm{~A}$ series of additional analyses were conducted for verification purposes. The reference model for the model comparison was created from the principal components obtained from an exploratory factorial analysis involving the eight experimental factors. We report the procedure, the R script and the results in Appendix C .
} 
ratio of the two a posteriori distributions was used to obtain the $\mathrm{BF}_{10}$, as well as the $\mathrm{BF}_{\text {hyp }}$ when the $\mathrm{BF}_{10}$ was greater than 3 . We applied this approach to both syllable frequency measures. Prior distribution choice and the analytical procedure were the same.

\section{RESULTS}

4500 handwriting reaction times (RT) were recorded. Values larger or smaller than the mean $\mathrm{RT} \pm 2.5$ standard deviations for each participant were excluded, i.e., 115 outlier values $(2.56 \%)$. Four categories of errors were identified: the participants made an orthographic error (araigné for araignée [spider]; 94 values, 2.09\%); they used an alternative name for a picture (livre [book] for agenda [diary]; 668 values, 14.84\%); they handwrote ToT (tip of the tongue, 103 values, 2.29\%); they handwrote DOK (“don't know”, 86 values, 1.91\%). Analyses were therefore run on 3434 values.

We first specified the group-level effects part of the linear regression model. The posterior distribution of the correlations between group-level effects seemed to be rather widely distributed (Appendix A). Moreover, all estimated values were less than .14, i.e., less than $2.0 \%$ common variance. This suggested that arguments in the data for estimating correlations were relatively weak, i.e., there was little benefit in adding all correlations between group-level effects. As far as group-level standard deviations (by-participant adjustment on slopes) were concerned, there was little benefit in adding all group-specific coefficients (Appendix B). In the final model, we included group-level effects only on Intercept for participants and for items. Appendix A and B are available on OSF (http://osf.io/ gazf3) 
Table 2. Mean (Estimate) and standard deviations (Standard errors) of the posterior distribution, boundaries of the 95\% Credible Intervals, and the two Bayes Factors of the multiple regression model.

\begin{tabular}{crrrrrr}
\hline & Estimate & Est.Error & l-95\% CI & u-95\% CI & BF $_{10}$ & BF $_{\text {hyp }}$ \\
\hline Intercept & 1264.6 & 131.27 & 1000.4 & 1521.23 & $2.07 * 10^{16}$ & $+\infty$ \\
NA (h) & 108.63 & 29.39 & 51.5 & 165.89 & 192.99 & 7999 \\
IA & -42.74 & 16.71 & -75.37 & -10.23 & 4.81 & 199 \\
Ivar & -69.96 & 22.36 & -115.08 & -26.44 & 45.63 & 1332 \\
AoA & 81.8 & 21.03 & 41.06 & 123.38 & 359.8 & $+\infty$ \\
LogFreq & -29.1 & 25.43 & -79.79 & 20.15 & .42 & - \\
NbLett & -6.48 & 6.75 & -19.66 & 7.02 & .09 & - \\
Fam & 28.93 & 12.77 & 3.58 & 53.95 & 1.59 & - \\
VC & 18.88 & 12.85 & -6.98 & 43.89 & 0.36 & - \\
\hline
\end{tabular}

Note. $N A(h)=$ Name Agreement, h-statistic measures; IA = Image Agreement; Ivar = Image Variability; AoA = Age of Acquisition; LogFreq = Natural logarithm of lexical frequency; NbLett $=$ Number of letters; Fam = Conceptual Familiarity; VC= Visual Complexity

The multiple linear regression model was similar to those reported in the literature (Table 2). The likelihood of the model inclusing of the eight experimental factors was $3.05 * 10^{12}$ given the observed data greater than the likelihood of the model without any experimental factors given the observed data. Together, all the eight factors accounted for $41.86 \%$ of the variance. Two factors appeared to be important predictors of reaction times (Table 2): the age at which a word is learnt (AoA) and name agreement $(\mathrm{hNA})$. The $\mathrm{BF}_{10}$ favoured the model that included the two factors. Moreover, the $\mathrm{BF}_{\text {hyp }}$ favoured the expected effect direction for reaction times (Figure 2). Imageability (Ivar) also influenced reaction times. The $\mathrm{BF}_{10}$ indicated that the likelihood of the hypothesis $\beta \neq 0$ given the observed data was about 46 times greater than the 
hypothesis $\beta=0$. The $\mathrm{BF}_{\text {hyp }}$ favoured the expected negative relationship between image variability and initialisation latencies (Figure 2$)$. The value $\mathrm{P}\left(\beta \neq 0 \mid \mathrm{D}_{\text {ata }}\right.$ ) was about 5 times greater than that of $\mathrm{P}\left(\beta=0 \mid \mathrm{D}_{\text {ata }}\right)$ for Image Agreement. Moreover, the $\mathrm{BF}_{\text {hyp }}$ for this factor was in favour of the expected negative relation (Figure 2), Finally, the $\mathrm{BF}_{10}$ for Lexical Frequency, Familiarity, Visual Complexity and Number of Letters were in favour of the model not including these factors, suggesting that these three factors are not predictors of handwritten naming latencies. The reference model used to test the syllable frequency effect therefore includes four factors: Age of Acquisition, Image Variability and Image Agreement and Name Agreement.

Figure 2. Graphical representation of the relationship between each of the eight experimental factors and handwritten naming latencies.

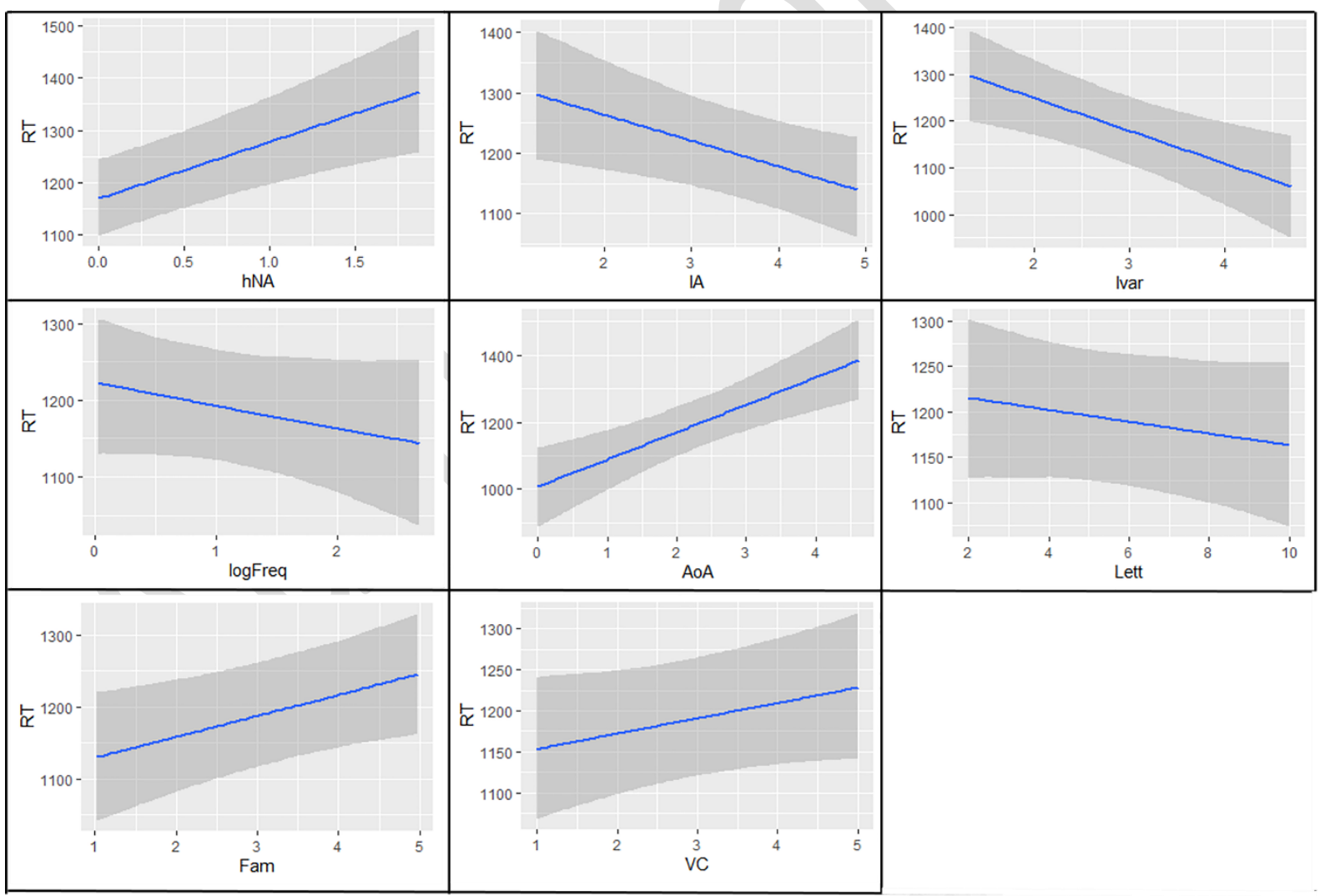

First, syllable frequency was operationalised based on the average of the syllable frequencies. The group-level standard deviation (by-participant adjustment on syllable frequency slope) was relatively small $(s d=3.46)$. It was not included in the model. The 
estimation of the syllable frequency effect was rather small $(\beta=-16.98, \mathrm{sd}=13.98,95 \%$-CI $\{-$ $43.38,8.66\})$. The likelihood of $\mathrm{H}_{1}(\beta \neq 0)$ given the observed data was 0.34871 times greater than the likelihood of $\mathrm{H}_{0}(\beta=0)$ given the observed data. In other words, $\mathrm{P}\left(\beta=0 \mid \mathrm{D}_{\text {ata }}\right)$ was about 3 times more likely than $\mathrm{P}\left(\beta \neq 0 \mid \mathrm{D}_{\text {ata }}\right)$, thus suggesting that syllable frequency is not a predictor of handwritten naming latencies.

As far as the first syllable frequency effect was concerned, the group-level standard deviation (by-participant adjustment on first syllable frequency slope) was not included in the model $(\mathrm{sd}=.3 .89)$. As for the mean syllable frequency effect, the estimation of the first syllable frequency effect was rather small $(\beta=-13.26, \mathrm{sd}=10.95,95 \%-\mathrm{CI}\{-34.55,7.94\})$. The $\mathrm{BF}_{10}=$ .00018 favoured the $\mathrm{H}_{0}$, i.e., the likelihood of the model not including the first syllable frequency $\left(\mathrm{H}_{0} \beta=0\right)$ given the observed data was about 5556 times greater than the likelihood of the model including this factor $\left(\mathrm{H}_{1} \beta \neq 0\right)$.

\section{DISCUSSION}

The aim of the study presented here was to test whether Kandel et al.'s (2011) hypothesis that access to the abstract orthographic wordform involves a syllabic level situated between the lexical and letter representations. If orthographic syllables are stored in O-LTM, their frequency of occurrence in a language should impact the efficiency with which this representation is retrieved. Empirically, this should result in a shorter preparation time for the handwritten verbal response (i.e. initialisation latencies) for words composed of high-frequency syllables than for those containing low-frequency syllables. We explored this hypothesis using a Bayesian approach. The latter choice was motivated by the fact that it was possible to specify the likelihood of either the presence $(\mathrm{H} 1)$ or absence $(\mathrm{H} 0)$ of an influence of experimental factors (Perret \& Bonin, 2019). First, a simultaneous multiple regression analysis was performed with eight experimental factors (Bonin et al., 2002; Perret \& Laganaro, 2013; Perret \& Bonin, 2019). Our aim was to obtain a reference model to test the syllabic frequency effect. Second, the 
posterior distributions of the models with and without syllable frequency measures were compared to compute Bayes Factors. Two measures of syllable frequency were used, one corresponding to the mean of the syllable frequency and another one corresponding to the first syllable frequency.

With regard to the linear regression analysis, the pattern of results was similar to the one reported in the literature (Bonin et al., 2002; Perret \& Laganaro, 2013; Perret \& Bonin, 2019). Name and Image Agreements (hNA \& IA), Imaging Variability (Ivar) and Age of Acquisition $(\mathrm{AoA})$ were determinants of handwritten latencies. The Bayes Factors $\left(\mathrm{BF}_{10}\right)$ indicated that the likelihood of the model including of each of these three factors $\left(\mathrm{H}_{1}\right)$ given the observed data was greater (Table 2) than the model without their inclusion $\left(\mathrm{H}_{0}\right)$. Furthermore, the $\mathrm{BF}_{\text {hyp }}$ supported the expected direction of the relationship between each of these three factors and the latencies. Finally, the $\mathrm{BF}_{10}$ for the other four factors (Visual Complexity, Conceptual Familiarity, Number of letters and Lexical Frequency) indicated that the likelihood of the model not including these factors $\left(\mathrm{H}_{0}\right)$ given the observed data was greater than the model including these factors $\left(\mathrm{H}_{1}\right)$.

Once the influence of the main determinants of written naming latencies for the images was removed, the analyses were in favour of an absence of syllable frequency effects. $\mathrm{BF}_{10}$ indicated that the likelihood of the model not including the mean syllable frequency $\left(\mathrm{H}_{0}\right)$ given the observed data was about 3 times greater than the model including this factor $\left(\mathrm{H}_{1}\right)$. This $\mathrm{BF}_{10}$ increased highly (5556) when first syllable frequency was used. It is important to note that using a Bayesian approach, we can argue in favour of $\mathrm{H}_{0}$ (Kass \& Raftery, 1995). The Bayesian approach makes it possible to estimate the likelihood of $\mathrm{H}_{0}$ and $\mathrm{H}_{1}$ given the observed data and thus indicate the presence or absence of an influence of syllable frequency ${ }^{9}$. From a theoretical

\footnotetext{
${ }^{9}$ The question of the choice of priors seems to be eliminated. The Bayesian analysis implies choosing a prior distribution and its characteristics, i.e., prior distribution of probability. The choice of this distribution is important because it has a direct impact on the results of estimates of posterior distributions (e.g., Kass \& Raftery, 1995). We therefore performed the analyses for syllable frequency with three different prior
} 
point of view, this absence of influence constitutes an argument against the hypothesis that syllables are accessed in O-LTM during the encoding of the orthographic wordform. As discussed in the Introduction, this hypothesis of an orthographic mental syllabary is attractive because it accounts for all the results reported in the literature (e.g., Caramazza \& Miceli, 1990; Hess et al., 2019; Kandel et al., 2006; Lambert et al., 2008; Sausset et al., 2012). The challenge arising from our results is to account for this literature on syllables while finding an alternative to the conception proposed by Kandel et al (2011).

Inspiration could perhaps come from work on wordform encoding in spoken production. In a similar way to Kandel et al. (2011), Dell (1986; Mackay, 1987) described a conception of access to phonological representation which includes a syllabic level between lexical representations and phonological segments. The objective was to account for the constraints that syllabic structure imposes on the types of errors made in spoken production (e.g., Dell, 1986; Fromkin, 1971; Mackay, 1972; Shattuck-Hufnagel, 1979). However, it soon became clear that this conception did not make it possible to account for the results reported in the literature (see Roelofs, 1997, 2000 for a comprehensive discussion of this issue). It is currently accepted that the syllabic information and segmental information are represented independently in the phonological mental lexicon. Even if several alternatives exist, particularly with regard to the characteristics of the syllabic structure (see Goldrick, 2014 for a review), the encoding of the phonological representation is conceived as involving, on the one hand, the parallel retrieval in memory of these two types of information and, on the other, a process aimed at associating each segment with a syllabic position (e.g., syllabification process). Phonological syllables are thus constructed "on-line" thanks to this incremental association process (Levelt,

distributions: a uniform distribution $(U[-100,100])$, a normal distribution $N[0,100]$ and a second normal distribution with a 10 times smaller standard deviation $(N[0,10])$. All three analyses gave similar results to those observed with the prior used for the analyses $(\mathrm{N}[0,100])$. 
Roelofs \& Meyer, 1999). Finally, some authors (e.g., Jacquemot \& Scott, 2006) have suggested that this syllabification process is linked to the phonological output buffer.

It seems possible to transfer this conception to access to orthographic representations by replacing retrieval in an orthographic mental syllabary (Kandel et al., 2011, Figure 1) with a process of "on-line" construction of syllables. This proposal implies making the hypothesis that the lexical representation active from the O-LTM in parallel and independently the orthographic segmental information and syllabic information. A syllabification process then associates these two types of information. The orthographic syllables are thus built sequentially and stored in O-WM. The advantage of this adaptation is its ability to reconcile the absence of syllable frequency effects reported here with all the data reported in the literature on the role of the syllable in handwritten word production. On the one hand, since syllables are constructed and not retrieved, their frequency of occurrence in a language should not impact the efficiency of orthographic wordform encoding. No influence of syllable frequency on the preparation time of the handwritten verbal response is therefore predicted. On the other hand, the result of orthographic encoding is a representation with a syllabic organisation in O-WM. An influence of the syllabic structure on the performance of patients with an impaired graphical buffer (Badecker, 1996; Caramazza \& Miceli, 1990) is therefore expected. This also applies to the influence of the number of syllables on the latencies for second and third copies (Lambert et al., 2008), as the refresh process that keeps the orthographic representation active in O-WM can still be based on syllables. Since the central and peripheral levels process the information in parallel (Kandel et al., 2011; Olive, 2014; Roux et al., 2013), we can assume that the "on-line" construction for the post-boundary syllable takes place at the point when the boundary is crossed. At the empirical level, the consumption of attentional resources due to this syllabification process results in an increase in the duration of the inter-letter interval (e.g., Alvarez et al., 2009; Hess et al., 2019; Kandel et al. 2006; Sausset et al., 2012, 2013). Finally, 
modulations of the influence of syllabic boundary and syllable number effects on second and third copy latencies reported by Sausset et al. (2012) are expected. When peripheral processing requires few resources, all syllables are refreshed before copying, thus accounting for the influence of the number of syllables. Alternatively, the syllables are constructed one after the other when peripheral processes are more resource-intensive (e.g. writing without visual feedback). A slowing-down of the trace then appears when crossing the syllabic boundary.

In summary, the aim of this work was to test the hypothesis that syllabic representations are stored in a syllabary and mobilised during the encoding of the orthographic verbal form (Kandel et al., 2011). The analyses reported here make it possible to reject this hypothesis. We propose an alternative design developed on the basis of spoken production studies (Goldrick, 2014). This proposal is based on the parallel and independent retrieval in long-term orthographic memory of the segmental and syllabic information of the word to be produced. A syllabification process would then associate these two pieces of information in order to construct each syllable "on-line". Finally, this syllable is kept active thanks to orthographic working memory. This concept has the advantage of being able to account for both the absence of an influence of syllable frequency on latencies and all the data in the literature on the syllable in handwritten production. As, to our knowledge, this is the first work on the influence of syllable frequency, it seems important to replicate it, especially in languages other than French. It also opens some specific questions concerning the format of syllabic information. A wide range of hypotheses have been developed for spoken production, from a version of the abstract frame coding only the number of syllables (Levelt et al., 1999) to a structure coding the Consonant/Vowel status and the syllabic position of each CV element for each word (Sevald, Dell \& Cole, 1995). Future work will have to specify the format of the abstract framework involved in orthographic encoding and its relationship to phonological processes. Finally, we note the importance of the use of the Bayesian approach. By making it possible to draw 
conclusions about the likelihood of observing the data given $\mathrm{H}_{0}$, these analyses open up the possibility of constraining the development of theoretical models by excluding the absence of certain factors. 
Acknowledgement: This research received no specific grant from any funding agency in the public, commercial, or not-for-profit sectors. The authors wish to thank Patrick Bonin and Sonia Kandel for the scientific exchanges on this work and two anonymous reviewers for very helpful comments on a previous version of this paper.

Disclosure Statement: No potential conflict of interest was reported by the author(s). 


\section{References}

Afonso, O. \& Alvarez, C. J. (2011). Syllable frequency effects in Spanish handwriting production. Logopedia, Foniatria y Audiologia, 31, 33-38.

Alario, F-X. \& Ferrand, L. (1999). A set of 400 pictures standardized for French: Norms for name agreement, image agreement, familiarity, visual complexity, image variability, and age of acquisition. Behavior Research Methods, Instruments, and Computers, 31, 531-552.

Alvarez, C. J., Cottrell, D., \& Afonso, O. (2009). Writing dictated words and picture names: Syllabic boundaries affect execution in Spanish. Applied Psycholinguistics, 30, 205-223.

Badecker, W. (1996). Representational proprieties common to phonological and orthographic output systems. Lingua, 99, 55-83.

Bogaerts, H., Meulenbroek, R. G. J., \& Thomassen, A. J. W. M. (1996). The possible role of the syllable as a processing unit in handwriting. In M. L. Simner, C. G. Leedham, \& A. J. W. M. Thomassen (Eds.), Handwriting and drawing research: Basic and applied issues (pp. 115-126). Amsterdam: IOS Press.

Bonin, P., Fayol, M., \& Chalard, M. (2001). Age of acquisition and word frequency in written picture naming. The Quarterly Journal of Experimental Psychology A, 54(2), 469-489.

Bonin, P., Fayol, M., \& Gombert, J.-E. (1998). An experimental study of lexical access in the writing and naming of isolated words. International Journal of Psychology, 33, 269-286.

Bonin, P., Laroche, B., \& Perret, C. (2016). Locus of word frequency effects in spelling to dictation: still at the orthographic level! Journal of Experimental Psychology: Learning, Memory and Cognition, 42, 1814-1820.

Bonin, P., Chalard, M., Méot, A. \& Fayol, M. (2002). The determinants of spoken and written picture naming latencies. British Journal of Psychology, 93, 89-114.

Bonin, P., Méot, A., Lagarrigue, A, \& Roux, S. (2015). Written object naming, spelling to dictation, and immediate copying: Different tasks, different pathways? The Quarterly Journal of Experimental Psychology, 68, 1268-1294.

Bonin, P., Peereman, R., Malardier, N., Méot, A. \& Chalard, M. (2003). A new set of 299 pictures for psycholinguistic studies: French norms for name agreement, image agreement, conceptual familiarity, visual complexity, image variability, age of acquisition and naming latencies. Behavior Research Methods, Instruments, \& Computers, 35, 158-167.

Bürkner P. C. (2017). brms: An R Package for Bayesian Multilevel Models using Stan. Journal of Statistical Software, 80, 1-28.

Bürkner P. C. (2018). Advanced Bayesian Multilevel Modeling with the R Package brms. The R Journal, 10, 395-411.

Caramazza, A., \& Miceli, G. (1990). The structure of graphemic representations. Cognition, 37, 243-297.

Caramazza, A., Miceli, G., Villa, G., \& Romani, C. (1987). The role of the Graphemic Buffer in spelling: Evidence from a case of acquired dysgraphia. Cognition, 26, 59-85.

Chetail, F., \& Mathey, S. (2010). InfoSyll: A syllabary providing statistical information on phonological and orthographic syllables. Journal of Psycholinguistic Research, 39, 485-504.

Dell, G.S. (1986). A spreading-activation theory of retrieval in sentence production. Psychological Review, 93, 283-321. 
Dienes, Z. (2016). How Bayes factors change scientific practice. Journal of Mathematical Psychology, 72, 78-89.

Ellis, A. W. (1988). Normal writing processes and peripheral acquired dysgraphias. Language and Cognitive Processes, 3, 99-127.

Forster, K., I. \& Forster, J., C. (2003). DMDX: A windows display program with millisecond accuracy. Behavior Research Methods, Instruments, \& Computers, 35, 116-124.

Fromkin, V. A. (1971). The non-anomalous nature of anomalous utterances. Language, 27-52.

Gelman, A. (2006). Prior distributions for variance parameters in hierarchical models (comment on article by Browne and Draper). Bayesian Analysis, 1, 515-534.

Gelman, A., Goodrich, B., Gabry, J., \& Vehtari, A. (2019). R-squared for Bayesian Regression Models, The American Statistician, 73, 307-309.

Goldrick, M. (2014). Phonological processing: The retrieval and encoding of word form information in speech production. In M. Goldrick, V. Ferreira, \& M. Miozzo (Eds.), Oxford library of psychology. The Oxford handbook of language production (p. 228-244). Oxford University Press.

Hess, S., Mousikou, P., Verrel, J., Schroeder, S. (2019). Syllabic processing in handwritten word production in German children and adults. Human Movement Science, 65, 5-14.

Jacquemot, C., \& Scott, S. K. (2006). What is the relationship between phonological short-term memory and speech processing? Trends in Cognitive Sciences, 10, 480-486.

Jeffreys, H. (1961). Theory of probability (3rd ed.). New York, NY: Oxford University Press.

Jonsdottir, M. K., Shallice, T., \& Wise, R. (1996). Phonological mediation and the graphemic buffer disorder in spelling: Cross-language differences? Cognition, 59, 169-197.

Kandel, S., Alvarez, C. J., \& Vallée, N. (2006). Syllables as processing units in handwriting production. Journal of Experimental Psychology: Human Perception and Performance, 32, 19-31.

Kandel, S., Peereman, R., Grosjacques, G., \& Fayol, M. (2011). For a psycholinguistic model of handwritten production: Testing the syllable-Bigram Controversy. Journal of Experimental Psychology: Human Perception and Performance, 37, 1310-1322.

Kandel, S., \& Perret, C. (2015). How does the interaction between spelling and motor processes build up during writing acquisition? Cognition, 136, 325-336.

Kass, R. E., \& Raftery, A. E. (1995). Bayes factors. Journal of the American statistical association, 90, 773-795.

Kreiner, D., Price, R.Z., Gross, A.M. (2008). Linguistic boundaries as predictors of the time between letters in oral and typed spellings. The Journal of General Psychology, 135, $117-$ 130.

Kruschke, J. K. (2011). Bayesian assessment of null values via parameter estimation and model comparison. Perspectives on Psychological Science, 6, 299-312.

Laganaro, M., \& Alario, F.-X. (2006). On the locus of the syllable frequency effect in speech production. Journal of Memory and Language, 55, 178-196.

Lambert, E., Alamargot, D., \& Fayol, M. (2012). Why use a copy task to study spelling in handwriting? In M. Fayol, D. Alamargot, \& V. Berninger (Eds.) Translation of thought to 
written text while composing: advancing theory, knowledge, research methods, tools and Applications. Publisher Psychology Press.

Lambert, E., Sausset, S. \& Rigalleau, F. (2015). The ortho-syllable as a processing unit in handwriting: the mute e effect. Reading and Writing: An Interdisciplinary Journal, 28, 683698.

Lambert, E., Kandel, S., Fayol, M., \& Espéret, E. (2008). The effect of the number of syllables on handwriting production. Reading and Writing: An Interdisciplinary Journal, 21, 859-883.

Levelt, W. J. M., Roelofs, A., \& Meyer, A. S. (1999). A theory of lexical access in speech production. Behavioral and Brain Sciences, 22, 1-75.

MacKay, D. G. (1987). The organization of perception and action: A theory for language and other cognitive skills. New York: Springer.

MacKay, D. G. (1972). The structure of words and syllables: Evidence from errors in speech. Cognitive Psychology, 3, 210-227.

McCloskey, M., Badecker, W., Goodman-Schulman, R. A., \& Aliminosa, D. (1994). The structure of graphemic representations in spelling: Evidence from a case of acquired dysgraphia. Cognitive Neuropsychology, 11, 341-392.

New, B., Pallier, C., Brysbaert, M., \& Ferrand, L. (2004). Lexique 2: A new french lexical database. Behavior Research Methods, Instruments, \& Computers, 36, 516-524.

Olive, T. \& Kellogg, R.T. (2002). Concurrent activation of high- and low-level production processes in written composition. Memory and Cognition, 30, 594-600.

Olive, T. (2014). Toward an Incremental and Cascading Model of Writing: A review of research on writing processes coordination. Journal of Writing Research, 6, 173-194.

Perret, C., and Bonin, P. (2019). Which variables should be controlled for to investigate picture naming in adults? A Bayesian meta-analysis. Behavior Research Methods, 51, 2533-2545.

Perret, C. \& Laganaro, M. (2013). Why are written naming latencies (not) longer than spoken naming? Reading and Writing: An Interdisciplinary Journal, 26, 225-239.

Perret, C., Schneider, L., Dayer, G. \& Laganaro, M. (2014). Convergences and Divergences between neurolinguistic and psycholinguistic data in the study of phonological and phonetic encoding: A parallel investigation of syllable frequency effects in brain-damaged and healthy speakers. Language, Cognition and Neuroscience, 29, 714-727.

Perret, C. \& Olive, T. (Eds). (2019). Spelling and writing words: Theoretical and methodological advances. Boston/Leiden: Brill

Pinheiro, J. C., \& Bates, D. M. (2000). Mixed-effects models in S and S-PLUS. New York, NY: Springer. http://dx.doi.org/10.1007/978-1-4419-0318-1

Planton, S., Jucla, M., Roux, F. E., \& Démonet, J. F. (2013). The “'handwriting brain'. A metaanalysis of neuroimaging studies of motor versus orthographic processes. Cortex, 49, 27722787.

Purcell, J. J., Turkeltaub, P. E., Eden, G. F., \& Rapp, B. (2011). Examining the central and peripheral processes of written word production through a meta-analysis. Frontiers in Psychology, 2:1, doi:10.3389/fpsyg.2011.00239. 
Rapp, B., \& Fisher-Baum, S. (2015). Uncovering the Cognitive Architecture of Spelling. In A. E. Hillis (Éd.), The Handbook of Adult Language Disorders (2 éd., p. 59-86). Psychology Press.

Rapp, B., Purcell, J., Hillis, AE., Capasso, R., \& Miceli, G. (2016). Neural bases of orthographic long-term memory and working memory in dysgraphia. Brain, 139, 588-604.

Rapp, B., Epstein, C., \& Tainturier, M.-J. (2002). The integration of information across lexical and sublexical processes in spelling. Cognitive Neuropsychology, 19, 1-29.

Roelofs, A. (1997). Syllabification in Speech Production: Evaluation of WEAVER. Language and Cognitive Processes, 12, 657-694.

Roelofs, A. (2000). WEAVER++ and other computational models of lemma retrieval and wordform encoding. In L. Wheeldon (Ed.) Aspects of Speech Production. Sussex (UK): Psychological Press.

Roux, J.-S., McKeeff, T. J., Grosjacques, G., Afonso, O., \& Kandel, S. (2013). The interaction between central and peripheral processes in handwriting production. Cognition, 127, 235241.

Sausset, S., Lambert, E., Olive, T., \& Larocque, D. (2012). Processing of syllables during handwriting: Effects of graphomotor constraints. The Quarterly Journal of Experimental Psychology, 65, 1872-1879.

Sausset, S., Lambert, E., Olive, T., \& Larocque, D. (2013). Flexibility of orthographic and graphomotor coordination during a handwritten copy task: effect of time pressure. Frontiers in Pscyhology, 4:866. doi 10.3389/fpsyg.2013.00866.

Service, E., \& Turpeinen, R. (2001). Working memory in spelling: evidence from backward typing. Memory, 9, 395-421.

Sevald, C. A., Dell, G. S., \& Cole, J. S. (1995). Syllable structure in speech production: Are syllables chunks or schemas? Journal of Memory and Language, 34, 807-820.

Shattuck-Hufnagel, S. (1979). Evidence for a serial-ordering mechanism in sentence production. In W. E. Cooper \& E. C. Walker (Ed.) Sentence Processing: Psycholinguistic Studies Presented to Merrill Garrett. Hillsdale, New York: Erlbaum.

Snijder, T. A. B., \& Bosker, R. J. (1999). Multilevel analysis: An introduction to basic and advanced multilevel modeling. London, UK: Sage Publishers.

Stan Development Team. 2017. Stan Modeling Language Users Guide and Reference Manual, VERSION. https://mc-stan.org

Vandekerckhove, J., Rouder, J.N. \& Kruschke, J.K. (2018). Editorial: Bayesian methods for advancing psychological science. Psychonomic Bulletin and Review, 25, 1-4.

Van Galen, G. P. (1991). Handwriting: Issues for a psychomotor theory. Human Movement Science, 10, 165-191.

Vehtari, A., Gelman, A., and Gabry, J. (2017). Practical Bayesian model evaluation using leaveone-out cross-validation and WAIC. Statistics and Computing. 27, 1413-1432.

Ward, J., \& Romani, C. (2000). Consonant-vowel encoding and orthosyllables in a case of acquired dysgraphia. Cognitive Neuropsychology, 17, 641-663. 
Weingarten, R., Nottbusch, G., \& Will, U. (2004). Morphemes, syllables and graphemes in written word production. In T. Pechmann \& C. Habel (Eds.). Multidisciplinary Approaches to Language Production. (pp. 529-572). Berlin: Mouton de Gruyter.

Zesiger, P., Orliaguet, J. P., Boë, L. J., \& Mounoud, P. (1994). The influence of syllabic structure in handwriting and typing production. In C. Faure, G. Lorette, \& A. Vinter (Eds.), Advances in handwriting and drawing: A multidisciplinary approach (pp. 389-401). Paris: Europia.

Zhang, Q., \& Wang, C. (2014). Syllable frequency and word frequency effects in spoken and written word production in a non-alphabetic script. Frontiers in Psychology, 5:120, doi:10.3389/fpsyg.2014.00120. 
31

Figure Captions 